\title{
ON SOME FIXED POINT THEOREMS IN ABSTRACT DUALITY PAIRS
}

\author{
KINGA CICHOŃ, MIECZYSŁAW CICHOŃ, AND MOHAMED M. A. METWALI
}

\begin{abstract}
We study the existence of fixed points for some functional problems. We focus on bilinear operators which are extensions of the product of operators (Banaś-Dhage-type results) and obtain some results on abstract duality pairs (abstract triples). However, we do not restrict ourselves to these cases; in fact, some special cases which do no fit in the above description are indicated. In particular, our approach allows us to study some differential or integral problems, for which the operators are not acting on a fixed Banach space.
\end{abstract}

\section{INTRODUCTION}

A classical fixed point problem $x=K(x), x \in E$, is investigated in a large class of spaces $E$, including product of some spaces $([27,36])$ or in a form $x=H(x, x)$ with bilinear operators on $E \times E([34,3])$. Simultaneously, if $E$ is a Banach algebra, an operator $K$ can be investigated also in a form of a product ([5, 16, 17]), which allows to apply such results for quadratic problems. We recently solved the problem of the existence of fixed points for the case when $E=X \times Y$ with some pairs of spaces $(X, Y)$ for which the product of operators is in the third space (cf. [15]). However, for convolution integral equations $([9,31,36])$ it is not sufficiently investigated. At the same time, in many special cases $K$ is of a special form of a superposition of operators acting on different spaces. In this interesting case we can find a solution in different spaces; in particular, quadratic integral equations can be solved out of the class of Banach algebras (see [15], for instance).

In this paper we consider the general situation, i.e. not only for the pointwise product or the convolutions, but when $K$ is defined on a product of spaces $E_{1} \times E_{2}$. We follow the idea of abstract duality pairs (triples, in fact) from [43. Clearly, this operator should be a superposition with some operators acting on $E_{1}$ and $E_{2}$ with values in an expected space of fixed points $E$. The problem considered is more general than those considered in [5, 8, 15, 27] for compact operators on products of spaces.

2010 Mathematics Subject Classification. Primary 47H10; Secondary 47N20, 45G10.

Key words and phrases. fixed point theorem; function spaces; quadratic integral equations; bilinear operators. 
We stress the applicability of the problem considered for many different operators and triples of spaces $\left(E_{1}, E_{2}, E\right)$. Our approach allows us to solve the problem outside the recently investigated class of Banach algebras, i.e. when $(E, \otimes,+)$ is a Banach algebra, $E_{1}=E_{2}=E$, and $H(t, s)=t \otimes s$ for $t, s \in E$. Although the approach to quadratic problems via bilinear operators has a long history (see [34, 2, 3], for instance), it is not sufficiently investigated and the problems are studied separately for different bilinear operators. We will extend and unify existing results.

Our new general approach is motivated by some very recent studies of the problem of the abstract duality pairing of spaces (or abstract triples, see Swartz [43]). Although this concept was investigated till now with different goals (see [41, 42]), it seems to be very convenient in our study. We extend both the theory of fixed points and the research about abstract duality pairing provided in 43 .

We will study some fixed point problems of the form

$$
x=H(F(x), G(x)),
$$

where $F: Z \rightarrow X, G: Z \rightarrow Y$ are nonlinear operators and $H(\cdot, \cdot)$ is a bilinear operator $H: X \times Y \rightarrow Z$, where $X, Y, Z$ are some Banach spaces. $H$ is defined through its section operators $H_{x}=H(x, \cdot): Y \rightarrow Z$ and $H_{y}(\cdot, y): X \rightarrow Z$ which are required to be linear and continuous for all (fixed) $x \in X, y \in Y$ (cf. [8, 35]). As $X, Y$ are metrizable complete spaces, $H$ is jointly continuous ([35, Theorem 2.17]).

Our general approach allows us to cover the cases when operators $F$ and $G$ have values in some "intermediate" spaces (clearly, it is a less restrictive assumption than $X=Y=Z$ ). We will present also some special cases of particular interest in applications.

First, let us consider the simplest case: $X=Y=Z$ and $H(t, s)=s \cdot t$ the product (pointwise multiplication) of two, possibly nonlinear, operators $A \cdot B$ in the Banach algebra $C(I, \mathbb{R})$. In this case we cover some results by Banaś and Lecko [5] or Dhage [16]. However, let us mention two different important Banach algebras which are not covered in these papers, namely the space $G(I, \mathbb{R})$ of regulated functions and the space $B V(I, \mathbb{R})$ of functions with bounded variation (see [38]). We will collect many interesting examples in Section 2 but examples of such triples of spaces (called abstract duality pairs or abstract triples) can also be found in [43. Ch. 1].

This approach will simplify the choice of appropriate (usually different) spaces $(X, Y, Z)$ for quadratic integral equations as in [13]. Motivated by the study of such integral equations in the case of discontinuous solutions a basic fixed point theorem was proved in [15] for the special case of the product of two operators acting on some triple of regular ideal spaces. Some examples of such triples were presented by obtaining an extension for the Banaś theorem for Banach algebras $X=Y=Z=C(I, \mathbb{R})$ (see also [16, 17], for instance). Here we study a general case of operators $H: X \times Y \rightarrow Z$ satisfying some assumptions and covering the case of a pointwise product. Many examples of such mappings will be presented below.

We do not concentrate only on a unification of earlier theorems, but we extend the existing results and we will try to stress on some new important motivations. 
As corollaries, we will present some new results, we extend the existing ones and we indicate a new approach for the problems, where the operators considered are not acting on the same spaces. The possibility of applications of such a fixed point theorem for integral equations let us pay special attention to specific cases of operators, i.e. the pointwise product and the convolution of functions.

The paper is complemented by some examples, special cases of main results, and by a discussion about our assumptions.

\section{Special CASEs. Examples}

We start by recalling some interesting cases of bilinear forms and triples of Banach spaces which are discussed in this paper. We should present some representative examples that can be considered as important special cases. In this paper, we restrict ourselves to presenting as corollaries of the main theorem only some previously proved theorems. The future extensions are numerous and are based on the examples presented below.

Recall that a mapping $H: X \times Y \rightarrow Z$ is bilinear if it is linear in each variable. Let $I$ denote a compact interval in $\mathbb{R}$. Consider the following triples of spaces $(X, Y, Z)$ with an appropriate operator $H$. Note here that we will always require the boundedness of $H$ : $\|H(s, t)\|_{Z} \leq a \cdot\|s\|_{X} \cdot\|t\|_{Y}$ for some $a>0$. As usual, the norm of the bilinear operator $H$ is defined to be

$$
\|H\|=\inf \left\{a>0:\|H(x, y)\|_{Z} \leq a \cdot\|x\|_{X} \cdot\|y\|_{Y}, \text { for all }(x, y) \in X \times Y\right\},
$$

i.e. $\|H\|=\sup _{\|x\|_{X} \leq 1,\|y\|_{Y} \leq 1}\|H(x, y)\|_{Z}$.

(1) The choice of $X=Y=Z$ for any Banach algebra (the spaces of continuous functions $C(I)$, regulated functions $G(I)$, or functions with bounded variation $B V(I)$ on a compact interval $I$ - or some of their subalgebras, for instance) is good enough for $H(x, y)=x \cdot y$ (the pointwise multiplication, i.e. $(x \cdot y)(t)=x(t) \cdot y(t))$.

Thus a special case of our equation 1.1 is of the form

$$
x(t)=\lambda u(t, x(t)) \cdot \int_{a}^{b} K(t, s) f(s, x(s)) d s,
$$

considered in [7, 10] with solutions in the space $C(I)$, i.e. in the case $X=$ $Y=Z=C(I)$.

(2) For discontinuous functions, let us recall the Hölder inequality for Lebesgue spaces: $\|x \cdot y\|_{L^{1}} \leq\|x\|_{L^{p}} \cdot\|y\|_{L^{q}}$ whenever $\frac{1}{p}+\frac{1}{q}=1$. Thus, a triple

$$
(X, Y, Z)=\left(L^{p}(I), L^{q}(I), L^{1}(I)\right)
$$

is a bilinear triple.

Our approach allows us to study the problem 2.1 with solutions in Lebesgue spaces under a very general set of assumptions (cf. [14, for instance). 
(3) The product of two functions from an Orlicz space $x, y \in L_{M}(I)$ needs not be in $L_{M}(I)$ (it can even be not integrable). However, if $x$ and $y$ belong to some particular Orlicz spaces, then the product $x \cdot y$ belongs to a third Orlicz space. However, there exist Orlicz spaces such that if $u \in L_{U}(I)$ and $v \in L_{V}(I)$, then the product $u \cdot v$ belongs to some Orlicz space $L_{W}(I)$, i.e. such a triple

$$
(X, Y, Z)=\left(L_{U}(I), L_{V}(I), L_{W}(I)\right)
$$

is good enough with the pointwise product too. For some necessary and sufficient conditions for functions generating such Orlicz spaces we refer to [26. Lemma 13.5]. A simple consequence of the Hölder inequality is that the triple $(X, Y, Z)=\left(L_{U}(I), L_{\tilde{U}}(I), L^{1}(I)\right)$ is also a bilinear triple for $\tilde{U}$, the Orlicz function conjugated to $U$. This triple is also considered with the pointwise product $H$.

In such a case we are able to study the quadratic integral equation (2.1) having solutions in Orlicz spaces (i.e. with nonlinear growth of the operators being considered); see [13, 14, for instance.

(4) Sometimes, we need to consider a special case for $Y=L^{\infty}$ and some Köthe function spaces (or ideal spaces) $X=Z=E$, i.e. for which $\|x \cdot y\|_{E} \leq$ $\|x\|_{E} \cdot\|y\|_{L^{\infty}}$. The class of such spaces is described in [45, p. 66] and in [46]:

$$
(X, Y, Z)=\left(E, L^{\infty}(I), E\right) .
$$

This special case allows, in particular, to unify the proofs for quadratic and standard integral equations (put $u(t, x(t))=\rho=$ const. in (2.1)). See also [14] for some applications in Lebesgue or Orlicz spaces.

(5) Let us mention that the general study for $H(t, s)=t \cdot s$ in the case when $X$ and $Y$ are Köthe function spaces (ideal spaces) is presented in [25]. In particular, some sufficient conditions ensuring that the space $Z$ of pointwise products $z=x \cdot y$ of elements $x \in X$ and $y \in Y$ is still a Banach space are presented.

(6) Let $1 / r=1 / p+1 / q-1, p, q, r \geq 1$. Then the triple

$$
(X, Y, Z)=\left(L^{p}(I), L^{q}(I), L^{r}(I)\right)
$$

due to the Young inequality $\|x * y\|_{L^{r}} \leq A \cdot\|x\|_{L^{p}}\|y\|_{L^{q}}$ is a bilinear triple with the convolution $H(x, y)=x * y$. For a similar property of Orlicz or Lorentz spaces see [30] (cf. the Young-O'Neil inequality [32]).

It should also be pointed out that in the case of Lebesgue spaces or $C(I)$ and $X=Y=Z, H(t, s)=t * s$ and $F=G=$ Id the operator defined by the right-hand side of $(1.1)$ is the autoconvolution operator on bounded intervals considered in [22], for instance.

The applicability of such type of results in the theory of convolution integral equations is presented in the book [39] or (in a context similar to our approach) in some papers by Blasco [9]. Finally, let us note that some interesting results of this kind can be found in [18]. 
(7) Since the Young inequality holds true also in Orlicz spaces: $\|x * y\|_{L_{W}} \leq$ $c \cdot\|x\|_{L_{U}}\|y\|_{L_{V}}$, an interesting consequence is that the triple

$$
(X, Y, Z)=\left(L_{U}(I), L_{V}(I), L_{W}(I)\right)
$$

with the convolution $H$ is good enough in our consideration (see [26] for the details).

Let us note here that we are also partially motivated by O'Neil [33] and his definition of convolution operators, [33, p. 308], which form also a special case of the operators $H$ considered here. Due to the Hardy-Littlewood theorem ([33, p. 317]) of Hölder type, fractional integral operators considered in [33] are also good enough for our purposes (see also its generalized form [33, Theorem 6.1]).

(8) Consider the triple $\left(X, Y, X \otimes_{\pi} Y\right)$ with the mapping $H: X \times Y \rightarrow X \otimes_{\pi} Y$, where $H$ is the projective tensor product (see [37] for a general theory or [26] for Orlicz spaces).

Let us recall that the special bilinear map $(x, y) \rightarrow x \otimes y$ acts as a universal bilinear map, in the following sense: every other bilinear map on $X \times Y$ factors through this one via a linear mapping on the tensor product. Indeed, for every bilinear map $H: X \times Y \rightarrow Z$, there exists a unique linear mapping $H^{*}: X \otimes Y \rightarrow Z$ such that $H(x, y)=H^{*}(x \otimes y)$ for all $x \in X$ and $y \in Y$. The projective norm $\|\cdot\|_{\pi}$ on $X \otimes Y$ is equal to $\|x\|_{X} \cdot\|y\|_{Y}$, so our condition is satisfied (but $X \otimes Y$ is an incomplete space, in general). However, in our theorems we need to put a completion of $X \otimes Y$ with this projective norm, but, on the other hand, such a kind of applications seems to be far from our original motivations.

(9) (Cf. [38) If $E_{1}$ and $E_{2}$ are Banach spaces, let us denote by $L\left(E_{1}, E_{2}\right)$ the Banach space of all bounded linear operators $A: E_{1} \rightarrow E_{2}$ with the uniform operator topology. Defining $H(A, x)=A x \in E_{2}$ for $A \in L\left(E_{1}, E_{2}\right)$ and $x \in E_{1}$ we obtain in a natural way the bilinear triple

$$
(X, Y, Z)=\left(L\left(E_{1}, E_{2}\right), E_{1}, E_{2}\right) .
$$

In this case, $\|H(A, x)\|_{E_{2}} \leq\|A\|_{L\left(E_{1}, E_{2}\right)}\|x\|_{E_{1}}$, which is related to some integral equations of Stieltjes type $([38])$.

(10) (Cf. [38]) Similarly, if $E_{1}, E_{2}$, and $E_{3}$ are Banach spaces then

$$
(X, Y, Z)=\left(L\left(E_{1}, E_{2}\right), L\left(E_{2}, E_{3}\right), L\left(E_{1}, E_{3}\right)\right)
$$

forms a bilinear triple with the natural bilinear form given by the composition $H(A, B)=A \circ B \in L\left(E_{1}, E_{3}\right)$ of operators $A \in L\left(E_{1}, E_{2}\right)$ and $B \in L\left(E_{2}, E_{3}\right)$. Clearly $\|h(A, B)\| \leq\|A\|\|B\|$, where the norms are taken in appropriate spaces.

(11) (Cf. [38]) If $E^{\prime}$ is the dual to the Banach space $E$ then

$$
(X, Y, Z)=\left(E, E^{\prime}, \mathbb{C}\right)
$$


is a bilinear triple with $H\left(x, x^{\prime}\right)=x^{\prime}(x)$ for $x \in E$ and $x^{\prime} \in E^{\prime}$. Also

$$
(X, Y, Z)=(E, \mathbb{R}, E), \quad(X, Y, Z)=(\mathbb{R}, E, E), \quad(X, Y, Z)=(E, \mathbb{R}, E)
$$

are bilinear triples with the map $H(r, x)=r x$ and $H(x, r)=r x$, respectively, where $r \in \mathbb{R}$ and $x \in E$.

(12) (Cf. [8]) Finally, let us recall also some classical bilinear operators acting on appropriate triples of spaces collected in [8] (see also [35, 43]). Let $X=Y=Z=C(0, T)$, but instead of the pointwise product we can consider a bilinear compact operator of the form $H(f, g)(x)=\int_{0}^{x} f(t) g(t) d t$ ([8, Example 3]). Then $\|H(f, g)\|_{\infty} \leq T \cdot\|f\|_{\infty} \cdot\|g\|_{\infty}$. If we consider $X$ with an $L^{1}$-norm, then it forms a normed but incomplete space (and $Y, Z$ with the supremum norm). Then this operator is not compact (however being separately compact), but bounded: $\|H(f, g)\|_{\infty} \leq T \cdot\|f\|_{1} \cdot\|g\|_{\infty}$. For more examples of interesting bilinear operators and triples of spaces we refer to [8, Examples 1-7].

Recall that more examples of triples of spaces can be found in [43, Ch. 1].

Example 2.1. Let us list some, considered previously, particular cases of quadratic or convolution integral equations with their applications:

a) Biology: model of spread of a disease (epidemic model considered by Gripenberg [23]):

$$
x(t)=k\left(P-\int_{-\infty}^{t} A(t-s) x(s) d s\right) \cdot \int_{-\infty}^{t} a(t-s) x(s) d s .
$$

b) Physics: kinetic theory of gases (Hu, Khavanin and Zhuang [24]):

$$
x(t)=a(t)+\left[f(t, x(t))+\int_{0}^{\infty} g(t, s) x(s) d s\right] \cdot \int_{0}^{\infty} h(t, s) K(s, x(s)) d s .
$$

c) Physics: convolution-type integral equations in statistical mechanics, the Percus-Yevick equation (see Nussbaum [31] or Rus [36] for a detailed study):

$$
x(t)=1+\lambda \int_{t}^{1} x(s) \cdot x(s-t) d s .
$$

d) Astrophysics: the Chandrasekhar equation (Chandrasekhar, Rus [36], Leonard and Mullikin [29, 28], Stuart [40], and many others):

$$
x(t)=1+x(t) \int_{0}^{1} \frac{t}{t+s} \psi(s) x(s) d s .
$$

\section{Fixed point theorems. Applications}

As clarified in the previous section, our equation (1.1) covers, in particular, quadratic integral equations, convolution equations or some operator equations, and thus the fixed point theorem applied to this problem will be useful in many cases.

We will assume in what follows that the operator $H$ is bounded ([8, 35]):

$$
\|H(s, t)\|_{Z} \leq a \cdot\|s\|_{X} \cdot\|t\|_{Y},
$$


for all $s \in X, t \in Y$, and for some positive $a \in \mathbb{R}$. Let us recall that all operators indicated in a previous section satisfy this condition with some constant $a$.

We are able to use directly the Schauder fixed point theorem in the case when $H$ is compact. The property of compactness of such bilinear operators was perfectly described in $[8$ and we will propose to study more general conditions.

If $X$ is a subset of a Banach space $E$, then $\bar{X}$ and conv $X$ denote the closure and convex closure of $X$, respectively. By $B(x, r)$ we denote a ball centered at $x$ with radius $r$. The standard algebraic operations on sets will be denoted by the symbols $k \cdot X$ and $X+Y$ (the Minkowski addition). Moreover, by $\mathcal{M}_{E}$ we denote the family of all nonempty and bounded subsets of $E$ and by $\mathcal{N}_{E}$ its subfamily consisting of all relatively compact subsets. We will use an axiomatic approach to the notion of a measure of noncompactness.

Definition 3.1 (4]). A mapping $\mu: \mathcal{M}_{E} \rightarrow[0, \infty)$ is said to be a measure of noncompactness in $E$ if it satisfies the following conditions:

(i) $\mu(X)=0 \Rightarrow X \in \mathcal{N}_{E}$.

(ii) $X \subset Y \Rightarrow \mu(X) \leq \mu(Y)$.

(iii) $\mu(\bar{X})=\mu(\operatorname{conv} X)=\mu(X)$.

(iv) $\mu(\lambda X)=|\lambda| \mu(X)$, for $\lambda \in \mathbb{R}$.

(v) $\mu(X+Y) \leq \mu(X)+\mu(Y)$.

(vi) $\mu(X \cup Y)=\max \{\mu(X), \mu(Y)\}$.

(vii) If $X_{n}$ is a sequence of nonempty, bounded, closed subsets of $E$ such that $X_{n+1} \subset X_{n}, n=1,2,3, \ldots$, and $\lim _{n \rightarrow \infty} \mu\left(X_{n}\right)=0$, then the set $X_{\infty}=$ $\bigcap_{n=1}^{\infty} X_{n}$ is nonempty.

The set of all sets for which $\mu(X)=0$ will be called the kernel of the measure $\mu$ and denoted by ker $\mu$. Clearly ker $\mu \subset \mathcal{N}_{E}$, but not every relatively compact set should be in $\operatorname{ker} \mu$. For $\mu(A)=\|A\|=\sup _{a \in A}\|a\|$ we have $\operatorname{ker} \mu=\{0\}$.

Let us recall a classical example: the Hausdorff measure of noncompactness $\beta_{H}(X)$ (see [4]) is defined as follows:

$\beta_{H}(X)=\inf \{r>0$ : there exists a finite subset $Y$ of $E$ such that $x \subset Y+B(0, r)\}$, where $X$ is an arbitrary nonempty and bounded subset of $E$. For more examples we refer the reader to [4. To distinguish between measures of noncompactness $\mu$ in different spaces (if necessary) we will indicate an appropriate space as an index, i.e. $\mu_{E}, \mu_{E_{1}}$, etc.

We will require that the measures of noncompactness used in the assumptions of the main theorem have an additional special property which indicates how bilinear operators transform the measure of noncompactness between the spaces. It is analogous to that property, which is known for linear operators (cf. [11]) and, in some sense, to the property $(\mathrm{m})$ considered for the product in [6] or for convolution in [19, Theorem 5.20]. Let us stress that, at least for the sum, the product, and the convolution, the property presented below was studied and its importance is clear. In particular, it is a basis for the study of bilinear operators as contractions with respect to measures of noncompactness and is a key point of the proof of our fixed point theorem. 
First, let us prove that the Hausdorff measure of noncompactness possesses this property.

Lemma 3.2. Assume that the operator $H: X \times Y \rightarrow Z$ is bilinear and bounded. Then there exist constants $b, c>0$ such that

$$
\beta_{Z}(H(A \times B)) \leq b \cdot \beta_{X}(A)+c \cdot \beta_{Y}(B)
$$

for all bounded subsets $A \subset X, B \subset Y$.

Proof. Consider on $X \times Y$ the following norm: $\|(x, y)\|=\|x\|+\|y\|$. Clearly, being bounded, the operator $H$ is continuous from $(X \times Y,\|(\cdot, \cdot)\|)$ to $Z$ (cf. [8]).

Take arbitrary bounded sets $A \subset X$ and $B \subset Y$. From the definition of $\beta_{Z}$, for arbitrary $\varepsilon>0$ there exists a finite set $K_{1} \subset X$ such that

$$
\left.A \subset K_{1}+B\left(0, r_{1}+\varepsilon\right)\right),
$$

where $r_{1}=\beta_{X}(A)$, and there exists a finite set $K_{2} \subset Y$ such that

$$
\left.B \subset K_{2}+B\left(0, r_{2}+\varepsilon\right)\right),
$$

where $r_{2}=\beta_{Y}(B)$. Thus $A \times B$ can be covered by $\left(K_{1} \times K_{2}\right)+B\left(0, r_{1}+r_{2}+2 \varepsilon\right)$. Indeed, let $s \in K_{1}$ and $t \in K_{2}$. Then

$H(s, B) \subset H\left(s, K_{2}\right)+\left(r_{2}+\varepsilon\right) \cdot H(s, B(0,1)) \subset H\left(K_{1} \times K_{2}\right)+\left(r_{2}+\varepsilon\right) \cdot H(s, B(0,1))$ and

$H(A, t) \subset H\left(K_{1}, t\right)+\left(r_{1}+\varepsilon\right) \cdot H(B(0,1), t) \subset H\left(K_{1} \times K_{2}\right)+\left(r_{1}+\varepsilon\right) \cdot H(B(0,1), t)$.

Finally

$$
H(A \times B) \subset H\left(K_{1} \times K_{2}\right)+\left(r_{1} \cdot\|B\| \cdot a+r_{2} \cdot\|A\| \cdot a+2 \varepsilon\right) \cdot B(0,1)
$$

and then

$\beta_{Z}(H(A \times B)) \leq r_{1} \cdot\|B\| \cdot a+r_{2} \cdot\|A\| \cdot a+2 \varepsilon \leq a \cdot\|B\| \cdot \beta_{X}(A)+a \cdot\|B\| \cdot \beta_{Y}(B)+2 \varepsilon$.

By passing to the limit with $\varepsilon \rightarrow 0^{+}$we get the expected inequality with $b=a \cdot\|B\|$ and $c=a \cdot\|A\|$, where $a$ is taken from (3.1.

Denote by $\alpha(B)$ the Kuratowski measure of noncompactness of a set $B([4)$. As $\beta(B) \leq \alpha(B) \leq 2 \cdot \beta(B)$, the same property holds true for the Kuratowski measure of noncompactness with $b=c=2$ (for the same norm on $X \times Y$ ). Similarly, it holds true also for measures of noncompactness like $\operatorname{diam}(\cdot)$ or $\|\cdot\|$. Since the property is independent of the conditions defining an abstract measure of noncompactness, it will be assumed separately (cf. [11] for the case of linear operators).

Thus, let us formulate the following condition:

Condition: Given a triple of spaces $(X, Y, Z)$, a bilinear operator $H: X \times Y \rightarrow Z$, and measures of noncompactness $\left(\mu_{X}, \mu_{Y}, \mu_{Z}\right)$, there exist constants $b, c>0$ such that $H$ satisfies

$$
\mu_{Z}(H(A, B)) \leq b \cdot \mu_{X}(A)+c \cdot \mu_{Y}(B)
$$

for all bounded subsets $A \subset X, B \subset Y$. 
For the case $H(t, s)=s \cdot t$ our condition coincides with the condition (m) considered in [6] or [19] (with $b=\|B\|$ and $c=\|A\|$ ) and where it was proved for some measures of noncompactness ([6, Theorem 2.3, Theorem 2.4]); cf. also the case of a convolution $H(t, s)=t * s$ in $L^{1}$ space in [19].

For the convenience of the reader, let us recall first a fixed point theorem due to Darbo, which will be useful in the proof of the main theorem.

Theorem 3.3 ([4]). Let $Q$ be a nonempty, bounded, closed, and convex subset of $E$ and let $H: Q \rightarrow Q$ be a continuous transformation which is a contraction with respect to the measure of noncompactness $\mu$ in $E$, i.e. there exists $\gamma \in[0,1)$ such that

$$
\mu(H(X)) \leq \gamma \cdot \mu(X),
$$

for any nonempty subset $X$ of $E$. Then $H$ has at least one fixed point in the set $Q$ and the set of all fixed points of $H$ belongs to $\operatorname{ker} \mu$.

Theorem 3.4. Consider the triple of Banach spaces $(X, Y, Z)$ with a bounded bilinear operator $H: X \times Y \rightarrow Z$, i.e. satisfying (3.1) and (3.2). Moreover, let $T$ be a nonempty, bounded, closed, convex subset of $Z$, and let $F: Z \rightarrow X$ and $G: Z \rightarrow Y$. Assume that

(F1) $F$ transforms continuously the set $T$ into $T_{1} \subset X$ and $F(T)$ is bounded in $X$;

(F2) there exists a constant $k_{1}>0$ such that $F$ satisfies $\mu_{X}(F(U)) \leq k_{1} \cdot \mu_{Z}(U)$ for any arbitrary bounded subset $U$ of $T$;

(G1) $G$ transforms continuously the set $T$ into $T_{2} \subset Y$ and $G(T)$ is bounded in $Y$;

(G2) there exists a constant $k_{2}>0$ such that $B$ satisfies $\mu_{Y}(G(U)) \leq k_{2} \cdot \mu_{Z}(U)$ for any arbitrary bounded subset $U$ of $T$;

(B) for every $x \in T_{1}$ and $y \in T_{2}$ we have $H(x, y) \in T$;

(S) constants b, $c$ in (3.2) satisfy the condition $b \cdot k_{1}+c \cdot k_{2}<1$.

Then there exists at least one fixed point for 1.1), i.e. there exists $x \in T$ such that

$$
x=H(F(x), G(x)),
$$

the set of all fixed points for this problem is relatively compact in $Z$, and it belongs to the kernel ker $\mu_{Z}$.

Proof. First, let us note that by (F1) and (G1) the operator $S: Z \rightarrow Z$ given by $S(z)=H(F(z), G(z))$ is well defined. Moreover, since for any $z \in T$ we have $F(z) \in T_{1}, G(z) \in T_{2}$, by (B) $S$ maps the set $T \subset Z$ into itself. Clearly, $S$ is bounded.

We need only to prove that the operator $S$ satisfies all conditions of the Darbo fixed point theorem (see 4, for instance).

Let $\left(x_{n}\right)$ be an arbitrary sequence in $T$ converging to $x \in T$. Let $\varepsilon>0$ be arbitrary. Despite the fact that $H$ needs not be uniformly continuous, its section operators are linear and continuous, so they are uniformly continuous. This implies the existence of $N_{1} \in \mathbb{N}$ such that $\left\|H\left(F\left(x_{n}\right), y\right)-H(F(x), y)\right\|_{Z} \leq \varepsilon / 2$ whenever 
$n \geq N_{1}, y \in Y$, and of $N_{2} \in \mathbb{N}$ such that $\left\|H\left(t, G\left(x_{n}\right)\right)-H(t, G(x))\right\|_{Z} \leq \varepsilon / 2$ whenever $n \geq N_{2}, t \in X$. Then

$$
\begin{aligned}
\left\|S\left(x_{n}\right)-S(x)\right\|_{Z}= & \left\|H\left(F\left(x_{n}\right), G\left(x_{n}\right)\right)-H(F(x), G(x))\right\|_{Z} \\
= & \| H\left(F\left(x_{n}\right), G\left(x_{n}\right)\right)-H\left(F(x), G\left(x_{n}\right)\right) \\
& \quad+H\left(F(x), G\left(x_{n}\right)\right)-H(F(x), G(x)) \|_{Z} \\
\leq & \left\|H\left(F\left(x_{n}\right), G\left(x_{n}\right)\right)-H\left(F(x), G\left(x_{n}\right)\right)\right\|_{Z} \\
& \quad+\left\|H\left(F(x), G\left(x_{n}\right)\right)-H(F(x), G(x))\right\|_{Z} \leq \varepsilon .
\end{aligned}
$$

Thus $S$ is sequentially continuous, so continuous on $T$. It remains to prove that it is a contraction with respect to some measure of noncompactness.

Take an arbitrary bounded set $A \subset Z$. Then $F(A)$ is bounded in $X$ and $G(A)$ is bounded in $Y$. Let us estimate $\mu_{Z} S(A)$. As the property $(3.2)$ is assumed, we get

$$
\begin{aligned}
\mu_{Z} S(A) & =\mu_{Z} H(F(A), G(A)) \leq b \cdot \mu_{X}(F(A))+c \cdot \mu_{Y}(G(A)) \\
& \leq b \cdot k_{1} \cdot \mu_{Z}(A)+c \cdot k_{2} \cdot \mu_{Z}(A)=\left(b \cdot k_{1}+c \cdot k_{2}\right) \cdot \mu_{Z}(A) .
\end{aligned}
$$

Thus we can apply the Darbo fixed point theorem for $S: T \rightarrow T$ and we get our thesis.

Remark 3.5. For completeness, let us present a comment about our assumptions. The condition (3.2) holds true, in particular, for any compact bilinear operator (cf. [8, Proposition 1] for a detailed characterization, [35]), but in this case we can apply the Schauder fixed point theorem on $Z$, so our theorem is its extension for noncompact operators $H$.

Contraction conditions (F2) or (G2) are satisfied when $F$ (or $G$, respectively) is the sum of compact and Lipschitz operators. Clearly, it is not the only case, and sometimes such a type of condition is verified directly (see [12, Lemma 1] for $Z=C(I)$ and $X=L^{1}(I)$, where $F$ is a solution operator for the $m$-accretive problem considered in [12], for instance)

In contrast to the case of Krasnoselskii's fixed point theorem, i.e. the sum of two (or more) operators (cf. 47] for the problem of the form $A x+B x+C x=x$ or 48. for the problem $A(x, x)+B(x, x)+C(x, x)=x)$, the product, as well as the general case of bilinear operators $H$, is not sufficiently investigated.

In the particular case $H(t, s)=t \cdot s$ we have some corollaries. If $X=Y=Z$, then we have the following fixed point theorem, proved by Banaś and Lecko.

Corollary 3.6 ([5]). Let $X$ be a Banach algebra. Assume that $T$ is a nonempty, bounded, closed, and convex subset of $X$, and the operators $A: T \rightarrow X$ and $B$ : $T \rightarrow X$ are continuous with $A(T), B(T)$ bounded in $X$. Moreover, assume that $H=A \cdot B$ transforms $T$ into itself. If

(1) there exists a constant $k_{1}>0$ such that $A$ satisfies $\mu_{X}(A(U)) \leq k_{1} \cdot \mu_{X}(U)$ for any bounded subset $U$ of $T$,

(2) there exists a constant $k_{2}>0$ such that $B$ satisfies $\mu_{X}(B(U)) \leq k_{2} \cdot \mu_{X}(U)$ for any bounded subset $U$ of $T$, 
(3) $\mu_{X}$ satisfies the condition $(m)$,

(4) $\|A(T)\|_{X} \cdot k_{2}+\|B(T)\|_{X} \cdot k_{1}<1$, then there exists at least one fixed point for the operator $H$ in the set $T$.

For a triple of spaces (see [15] for some particular cases), to the best of our knowledge, only one theorem is known and it can also be obtained as a special case of our theorem. In this case the condition 3.2 holds true for $b=\sup _{t \in T}\|F(t)\|_{X}$ and $c=\sup _{t \in T}\|G(t)\|_{Y}$.

Corollary 3.7 ([15], Theorem 4.1]). Assume that $T$ is a nonempty, bounded, closed, convex subset of a Banach space $Z$, and let $F: Z \rightarrow X$ and $G: Z \rightarrow Y$ be operators. Moreover, assume:

(1) $F$ transforms continuously the set $T$ into $T_{1} \subset X$ and $F(T)$ is bounded in $X$;

(2) there exists a constant $k_{1}>0$ such that $F$ satisfies $\mu_{X}(F(U)) \leq k_{1} \cdot \mu_{Z}(U)$ for any bounded subset $U$ of $T$;

(3) $G$ transforms continuously the set $T$ into $T_{2} \subset Y$ and $G(T)$ is bounded in $Y$;

(4) there exists a constant $k_{2}>0$ such that $G$ satisfies $\mu_{Y}(G(U)) \leq k_{2} \cdot c_{Z}(U)$ for any bounded subset $U$ of $T$;

(5) the triple of spaces $(X, Y, Z)$ is such that for any $x \in X$ and $y \in Y, x \cdot y \in Z$ and $\|x \cdot y\|_{Z} \leq k \cdot\|x\|_{X} \cdot\|y\|_{Y}$

(6) for every $x \in T_{1}$ and $y \in T_{2}$ we have $H(x, y) \in T$;

(7) $\sup _{t \in T}\|F(t)\|_{X} \cdot k_{2}+\sup _{t \in T}\|G(t)\|_{Y} \cdot k_{1}<1$.

Then there exists at least one fixed point for the equation $x=F(x) \cdot G(x)$ in the set $T$ and the set of all fixed points Fix $H$ belongs to the kernel ker $\mu_{Z}$.

Remark 3.8. For instance, if our conditions are fulfilled when the measure of noncompactness is $\mu_{Z}(A)=\|A\|$, then our results imply the uniqueness of the fixed point and that only a trivial solution is allowed.

The simplest example: by taking $F(x)=\frac{1}{2} \sin x, G(x)=\frac{1}{3} \arctan x$, and $H(t, s)=t \cdot s$ for $X=Y=Z=\mathbb{R}$, we obtain that $x=H(F(x), G(x))$, i.e. $\sin x \cdot \arctan x=6 x$ has only the trivial solution $\left(b=c=1, k_{1}=\frac{1}{2}, k_{2}=\frac{1}{3}\right)$.

Example 3.9. Let us recall that our theorem is applicable in the case of the convolution. Consider the following convolution equation:

$$
x(t)=\lambda \cdot \int_{0}^{t} \frac{1}{1+(x(s))^{2}} \cdot \arctan x(t-s) d s,
$$

where $\lambda=k \cdot l, k, l>0, x \in B(0, r) \subset C([0,1])$. Here $X=Y=Z=C([0,1])$ and $F: Z \rightarrow X$ is given by $F(x)(t)=\frac{k}{1+(x(t))^{2}}$ and $G: Z \rightarrow Y$ by $G(x)(t)=$ $l \cdot \arctan x(t)$. Obviously, $H(F, G)(t)=(F * G)(t)=\int_{0}^{t} F(s) \cdot G(t-s) d s$.

By the Titchmarsh theorem this equation has no trivial solution (this convolution can be the zero function if and only if at least one of the functions considered is zero, which is not the case), but we will show the existence of a (non-negative) solution on some ball $B(0, r) \subset C([0,1])$. 
Both operators $F$ and $G$ are composition operators acting on $C([0,1])$. Note that $G$ is Lipschitz with constant $l$, so the condition (G2) is satisfied with $k_{2}=l$. Moreover, $F$ is locally Lipschitz and on $T=B(0, r)$ satisfies the Lipschitz condition with constant $2 \cdot k \cdot r$. The condition (F2) is satisfied on $T$ with $k_{1}=2 \cdot k \cdot r$ (cf. [1]).

For the convolution, by the Young inequality we get (3.1) with $a=1$, so our Lemma 3.2 gives us the condition 3.2 of the form: $\beta_{Z}(A \times B) \leq r \cdot \mu_{X}(A)+r$. $\mu_{Y}(B)$.

Since we consider the equation on $T=B(0, r)$ we can easily verify the boundedness conditions. We have $\|G(x)\| \leq l \cdot \frac{\pi}{2}$ and $\|F(x)\| \leq k \cdot 1$ for any $x \in T$. Thus $F(T) \subset B(0, k)$ and $G(T) \subset l \cdot \frac{\pi}{2}$. Our conditions (F1) and (G1) are satisfied.

By the Young inequality (see (3.1)), $\|H(F, G)\| \leq\|F\| \cdot\|G\|$, so for any $x \in$ $B(0, r)$ we have $H(F(B(0, r)), G(B(0, r)))=H\left(B(0, k), B\left(0, l \cdot \frac{\pi}{2}\right)\right)$. Thus for any $t \in T_{1}=B(0, k)$ and $s \in T_{2}=B\left(0, l \cdot \frac{\pi}{2}\right)$ we have $\|H(t, s)\| \leq k \cdot l \cdot \frac{\pi}{2}$.

The condition (B) is satisfied whenever $k \cdot l \cdot \frac{\pi}{2} \leq r$, i.e. for a given $r$ we need to take sufficiently small constants $l$ and $k$. It remains to prove that the condition $(\mathrm{S})$ is satisfied for such constants. But this means that we expect that $2 k r^{2}+l r<1$ (for $k, l, r>0$ ). Indeed, for any $k, l>0$ this inequality is satisfied for $r \in\left(0, \frac{-l+\sqrt{l^{2}+8 k}}{4 k}\right)$ and finally

$$
\frac{\pi}{2} \cdot k \cdot l \leq r \leq \frac{-l+\sqrt{l^{2}+8 k}}{4 k}
$$

For an appropriate choice of constants $\left(k=\frac{1}{4}, l=1\right.$, and $r=\frac{1}{2}$, for instance) we can verify all the conditions of Theorem 3.4 , so our equation has a non-trivial solution (by the Titchmarsh theorem) and non-negative (as $H$ is a positive operator). Clearly, this equation has only an illustrative role and we are not restricted to the case of such simple functions.

Remark 3.10. In fact, the assumptions (F2) and (G2) seem to be the most restrictive in our main theorem. They are related to the continuity and compactness properties of $F$ and $G$ (a sum of compact and Lipschitz operators, for instance) or, sometimes, they are even equivalent with Lipschitz conditions (as in the case of superposition operators: see [1], for instance). We will be able to replace them by some conditions related rather to the growth conditions. This will be discussed later. In that case, when spaces are ideal (or Köthe function spaces), we are able to relax our assumptions on $F$ and $G$ provided that the domain $T$ is, additionally, compact in measure.

A normed space $(X,\|\cdot\|)$ of (classes of) measurable functions $x: I \rightarrow U$ ( $U$ is a normed space) is called pre-ideal if for each $x \in X$ and each measurable $y: I \rightarrow U$ the relation $|y(s)| \leq|x(s)|$ (for almost all $s \in I$ ) implies $y \in X$ and $\|y\| \leq\|x\|$. If $X$ is also complete, it is called an ideal space (see [46]). We will say that a set $T$ in an ideal space $X$ is compact in measure if it is compact in the topology of convergence in measure, i.e. as a subset of the space of all measurable functions $L^{0}(I)$ (see [46]). 
For any $\varepsilon>0$, let $c$ be the measure of uniform integrability of the set $X$ in an ideal function space $E$ on the compact interval $I$ (cf. [45, Definition 3.9]):

$$
c(X)=\limsup _{\varepsilon \rightarrow 0} \sup _{\operatorname{meas} D \leq \varepsilon} \sup _{x \in X}\left\|x \cdot \chi_{D}\right\|_{E},
$$

where $\chi_{D}$ denotes the characteristic function of a measurable subset $D \subset I$. As the norm is used in this definition, to distinguish between measures of uniform integrability $c$ in different spaces we put an appropriate index, e.g. $c_{X}$ or $c_{Y}$.

We will consider some subspaces $X, Y, Z$ of a space of $L^{0}(I)$ of measurable functions and if the spaces are ideal regular spaces, then we obtain the fixed point theorem under very natural conditions. We are interested in replacing conditions (F2) and (G2) by less restrictive ones. We extend these contraction conditions by using the measures of uniform integrability instead of the noncompactness ones.

We need to investigate the property $\sqrt{3.2}$ for the measure of uniform integrability.

Lemma 3.11. Let $X, Y, Z$ be ideal function spaces on $I=[0,1]$ with the Lebesgue measure. Assume that the operator $H: X \times Y \rightarrow Z$ is bilinear and the condition (3.1) is satisfied. Then

$$
c_{Z} H(A \times B) \leq a \cdot c_{X}(A) \cdot c_{Y}(B)
$$

for all bounded subsets $A \subset X, B \subset Y$.

Proof. Consider again on $X \times Y$ the norm $\|(x, y)\|=\|x\|_{X}+\|y\|_{Y}$. The bounded bilinear operator $H$ is continuous from $(X \times Y,\|(\cdot, \cdot)\|)$ to $Z$ (cf. [8]).

Take arbitrary bounded sets $A \subset X$ and $B \subset Y$. Take an arbitrary $\varepsilon>0$. Let $D$ be an arbitrary measurable subset of $I$ with meas $D \leq \varepsilon$. Denote by $\chi_{D}$ the characteristic function of the set $D$.

Let $z \in H(A \times B)$ be arbitrary. Then there exist $x \in A, y \in B$ such that $z=H(x, y)$. As $\left(H(x, y) \cdot \chi_{D}\right)(t)=\left(H\left(x \cdot \chi_{D}, y \cdot \chi_{D}\right)\right)(t)$ for and $t \in I$ and $Z$ is an ideal space, we have $\left\|H(x, y) \cdot \chi_{D}\right\|_{Z}=\left\|H\left(x \cdot \chi_{D}, y \cdot \chi_{D}\right)\right\|_{Z}$. Thus we can estimate:

$$
\begin{aligned}
\left\|z \cdot \chi_{D}\right\|_{Z} & =\left\|H(x, y) \cdot \chi_{D}\right\|_{Z}=\left\|H\left(x \cdot \chi_{D}, y \cdot \chi_{D}\right)\right\|_{Z} \\
& \leq a \cdot\left\|x \cdot \chi_{D}\right\|_{X} \cdot\left\|y \cdot \chi_{D}\right\|_{Y} .
\end{aligned}
$$

Since $\sup _{A \times B}\|H(x, y)\|_{Z}=\sup _{x \in A} \sup _{y \in B}\|H(x, y)\|_{Z}$, by taking the supremum over $x \in A, y \in B$ and sets $D$ with meas $D \leq \varepsilon$, and then by passing to the limit with $\varepsilon \rightarrow 0^{+}$we obtain:

$$
c_{Z}(H(A \times B)) \leq a \cdot c_{X}(A) \cdot c_{Y}(B)
$$

Before the formulation of the next theorem, let us indicate the difference between our results. We present some special operators $F$ fulfilling our conditions described below and used in the next theorem. Usually, they do not satisfy the previous condition (F2).

Consider the following assumption: for a given bounded subset $T \subset B(0, r) \subset X$ there exists a constant $k_{1}>0$ such that the operator $F: T \rightarrow X$ satisfies the 
condition

$$
c_{X}(F(U)) \leq k \cdot c_{Z}(U)
$$

for any bounded subset $U$ of $T$. We will call such an operator a $c_{X}-c_{Z}$ contraction with constant $k$.

Lemma 3.12 ([21, Lemma 2]). Let $Z=L_{q}(I), X=L_{p}(I)$, where $I=[0,1]$. Assume that a continuous operator $F: Z \rightarrow X$ satisfies the condition

$$
|F(x)(s)| \leq|b(s)|+d|x(s)|^{q / p},
$$

where $b(s) \in L_{p}(I)$ is a fixed function, and $k$ is a positive constant, for all $x$ from some ball $B(0, r)$. Then for any subset $U \subset B(0, r)$ the operator $F$ is a $c_{X}-c_{Y}$ contraction on $U$ with constant $k=d \cdot r^{q / p}$.

Let $M$ and $N$ be complementary $N$-functions, i.e. $N(x)=\sup _{y \geq 0}(x y-M(x))$, where $N:[0,+\infty) \rightarrow[0,+\infty)$ is continuous, even and convex with $\lim _{x \rightarrow 0} \frac{N(x)}{x}=$ $0, \lim _{x \rightarrow \infty} \frac{N(x)}{x}=\infty$, and $N(x)>0$ if $x>0(N(u)=0 \Longleftrightarrow u=0)$. The Orlicz class, denoted by $\mathcal{O}_{M}$, consists of measurable functions $x: I \rightarrow \mathbb{R}$ for which

$$
\rho(x ; M)=\int_{I} M(x(t)) d t<\infty .
$$

We shall denote by $L_{M}(I)$ the Orlicz space of all measurable functions $x: I \rightarrow \mathbb{R}$ for which

$$
\|x\|_{M}=\inf _{\varepsilon>0}\left\{\int_{I} M\left(\frac{x(s)}{\varepsilon}\right) d s \leq 1\right\} .
$$

Let $E_{M}(I)$ be the closure in $L_{M}(I)$ of the set of all bounded functions. Note that $E_{M} \subseteq L_{M} \subseteq \mathcal{O}_{M}$. The inclusion $L_{M} \subset L_{R}$ holds if and only if there exist positive constants $u_{0}$ and $a$ such that $R(u) \leq a M(u)$ for $u \geq u_{0}$ (see [26] for the detailed theory).

For Orlicz spaces we have the following result.

Lemma 3.13 ([15, Lemma 4.6]). Let $Z=L_{M}(I), X=E_{M_{1}}(I)$, where $I=[0,1]$. Assume that $F$ is a superposition operator generated by a Carathéodory function $u: I \times Z \rightarrow X$ and $i$ s continuous. Then an acting condition

$$
|u(t, x)| \leq b(t)+d M_{1}^{-1}\left[M\left(\frac{x}{r}\right)\right], \quad t \in I \text { and } x \in \mathbb{R}
$$

where $b \in E_{M_{1}}(I)$ and $r, d \geq 0$, implies that $F$ is a $c_{X}-c_{Y}$ contraction with constant $k=\frac{d}{r}$.

Clearly, compact operators satisfy (3.3) with constant $k=0$, but the class of such contractions is larger. If the operator takes bounded subsets of $E$ into the family of sets with equiabsolutely continuous norms in $E_{1}$, it will be called generalized improving (see [44]).

Lemma 3.14 ([15, Corollary 4.8]). If the operator $F$ is generalized improving, then it satisfies (3.3) with $k=0$.

Since we have already explained the reason, we are able to present a variant of the fixed point theorem for ideal spaces. 
Theorem 3.15. Consider the triple of regular ideal Banach spaces $(X, Y, Z)$ of functions on a compact interval $I \subset \mathbb{R}$ with a bounded bilinear operator $H: X \times$ $Y \rightarrow Z$, i.e. satisfying (3.1). Assume that $T$ is a nonempty, bounded, closed, convex, and compact in measure subset of a space $Z$, and let $F: Z \rightarrow X$ and $G: Z \rightarrow Y$ be operators. Moreover, assume:

(F1) $D$ transforms continuously the set $T$ into $T_{1} \subset X$ and $F(T)$ is bounded in $X$;

(F3) there exists a constant $k_{1}>0$ such that $F$ satisfies $c_{X}(F(U)) \leq k_{1} \cdot c_{Z}(U)$ for any bounded subset $U$ of $T$;

(G1) $G$ transforms continuously the set $T$ into $T_{2} \subset Y$ and $G(T)$ is bounded in $Y$;

(G3) there exists a constant $k_{2}>0$ such that $G$ satisfies $c_{Y}(G(U)) \leq k_{2} \cdot c_{Z}(U)$ for any bounded subset $U$ of $T$;

(B) for every $x \in T_{1}$ and $y \in T_{2}$ we have $H(x, y) \in T$;

(C2) either $a \cdot k_{1} \cdot\|G\| \cdot$ meas $I<1$ or $a \cdot k_{2} \cdot\|F\| \cdot$ meas $I<1$.

Then there exists at least one fixed point for the operator $S(\cdot)=H(F(\cdot), G(\cdot))$ in the set $T$ and the set of all fixed points belongs to the kernel of the measure $\beta_{Z}$, i.e. Fix $K \in \operatorname{ker} \beta_{Z}$.

Proof. As in the previous theorem, by (F1), (G1) the operator $S: Z \rightarrow Z$ given by $S(z)=H(F(z), G(z))$ is well defined, and by (B) it maps the set $T \subset Z$ into itself. Clearly, $S$ is also bounded.

The continuity of $S$ can be proved as in Theorem 3.7. Now, we need to investigate the contraction property with respect to the measure of uniform integrability $c_{Z}$.

Assume that $X$ is a nonempty subset of $T$ and let the fixed constant $\varepsilon>0$ be arbitrary. Then for an arbitrary $x \in X$ and for a set $D \subset I$ with meas $D \leq \varepsilon$ we obtain

$$
\begin{aligned}
\left\|S(x) \cdot \chi_{D}\right\|_{Z} & =\left\|H(F(x), G(x)) \cdot \chi_{D}\right\|_{Z}=\left\|H\left(F(x) \cdot \chi_{D}, G(x) \cdot \chi_{D}\right)\right\|_{Z} \\
& \leq a \cdot\left\|F(x) \cdot \chi_{D}\right\|_{Z} \cdot\left\|G(x) \cdot \chi_{D}\right\|_{Z} .
\end{aligned}
$$

For any non-negative real-valued functions $f$ and $g$ we have $\sup _{I}(f \cdot g) \leq \sup _{I} f$. $\sup _{I} g$. In view of $(\mathrm{C} 2)$ either $a \cdot k_{1} \cdot\|G\| \cdot$ meas $I<1$ or $a \cdot k_{2} \cdot\|F\| \cdot$ meas $I<1$. In the first case, by definition of $c_{Z}$ and by taking the supremum over all $x \in X$ and all measurable subsets $D$ with meas $D \leq \varepsilon$ we get

$$
c_{Z}(S(X)) \leq a \cdot c_{Z}(F(X)) \cdot\|G\| \cdot \text { meas } I,
$$

and in the second case,

$$
c_{Z}(S(X)) \leq a \cdot\|F\| \cdot \operatorname{meas} I \cdot c_{Z}(G(X)) .
$$

In any case, by assumptions (F3) and (G3) we obtain

$$
c_{Z}(S(X)) \leq \omega \cdot c_{Z}(X)
$$

where $\omega<1$. 
Because $X \subset T$ is a nonempty, bounded, and compact in measure subset of an ideal regular space $Z$ and $S(X) \subset T$, by [20, Theorem 1] $c_{Z}(X)=\beta_{Z}(X)$ and then

$$
\beta_{Z}(S(X)) \leq \omega \cdot \beta_{Z}(X) .
$$

The inequality obtained above, together with the properties of the operator $S$ and the set $T$ established before, allows us to apply the classical Darbo fixed point theorem for the Hausdorff measure of noncompactness $\beta_{Z}$. If we suppose that $\beta_{Z}($ Fix $S) \neq 0$, then $S=$ Fix $S$ implies $\beta_{Z}($ Fix $S)=\beta_{Z}(S)<\beta_{Z}(S)$, a contradiction. This completes the proof.

Recall that both fixed point theorems can be applied to the study of quadratic or convolution integral equations under very natural assumptions, but as claimed in Section 2, we are not restricted to those cases.

\section{REFERENCES}

[1] J. Appell and P. P. Zabrejko, Nonlinear superposition operators, Cambridge Tracts in Mathematics, 95, Cambridge University Press, Cambridge, 1990. MR 1066204

[2] I. K. Argyros, On a contraction theorem and applications, in Nonlinear functional analysis and its applications, Part 1 (Berkeley, Calif., 1983), 51-53, Proc. Sympos. Pure Math., 45, Amer. Math. Soc., Providence, RI, 1986. MR 0843548

[3] I. K. Argyros, Some sufficient conditions for finding a second solution of the quadratic equation in a Banach space, Math. Slovaca 38 (1988), no. 4, 403-408. MR 0978771

[4] J. Banaś and K. Goebel, Measures of noncompactness in Banach spaces, Lecture Notes in Pure and Applied Mathematics, 60, Marcel Dekker, New York, 1980. MR 0591679

[5] J. Banas and M. Lecko, Fixed points of the product of operators in Banach algebra, PanAmer. Math. J. 12 (2002), no. 2, 101-109. MR 1895774

[6] J. Banaś and L. Olszowy, On a class of measures of noncompactness in Banach algebras and their application to nonlinear integral equations, Z. Anal. Anwend. 28 (2009), no. 4, 475-498. MR 2550700

[7] J. Banaś and K. Sadarangani, Solutions of some functional-integral equations in Banach algebra, Math. Comput. Modelling 38 (2003), no. 3-4, 245-250. MR 2004993

[8] Á. Bényi and R. H. Torres, Compact bilinear operators and commutators, Proc. Amer. Math. Soc. 141 (2013), no. 10, 3609-3621. MR 3080183

[9] O. Blasco, Bilinear maps and convolutions, in Nuclear groups and Lie groups (Madrid, 1999), 45-55, Res. Exp. Math., 24, Heldermann, Lemgo, 2001. MR 1858142

[10] J. Caballero, A. B. Mingarelli and K. Sadarangani, Existence of solutions of an integral equation of Chandrasekhar type in the theory of radiative transfer, Electron. J. Differential Equations 2006, No. 57, 11 pp. MR 2226930.

[11] M. Cichoń, A point of view on measures of noncompactness, Demonstr. Math. 26 (1993), no. 3-4, 767-777 (1994). MR 1265840.

[12] M. Cichoń, Noncompact perturbations of $m$-accretive operators in general Banach spaces, Comment. Math. Univ. Carolin. 33 (1992), no. 3, 403-409. MR 1209283

[13] M. Cichoń and M. M. A. Metwali, On quadratic integral equations in Orlicz spaces, J. Math. Anal. Appl. 387 (2012), no. 1, 419-432. MR 2845761

[14] M. Cichoń and M. M. A. Metwali, Existence of monotonic $L_{\varphi}$-solutions for quadratic Volterra functional-integral equations, Electron. J. Qual. Theory Differ. Equ. 2015, No. 13, 16 pp. MR 3325916 
[15] M. Cichoń and M. M. A. Metwali, On a fixed point theorem for the product of operators, J. Fixed Point Theory Appl. 18 (2016), no. 4, 753-770. MR 3571304.

[16] B. C. Dhage, Remarks on two fixed-point theorems involving the sum and the product of two operators, Comput. Math. Appl. 46 (2003), no. 12, 1779-1785. MR 2018766

[17] B. C. Dhage and M. Kumpulainen, Nonlinear functional boundary value problems involving the product of two nonlinearities, Appl. Math. Lett. 21 (2008), no. 6, 537-544. MR 2412374.

[18] O. Diekmann, M. Gyllenberg and H. R. Thieme, Perturbing semigroups by solving Stieltjes renewal equations, Differential Integral Equations 6 (1993), no. 1, 155-181. MR 1190171

[19] S. Dudek, Fixed point theorems in Fréchet algebras and Fréchet spaces and applications to nonlinear integral equations, Appl. Anal. Discrete Math. 11 (2017), no. 2, 340-357. MR 3719830

[20] N. A. Erzakova, Compactness with respect to a measure, and a measure of noncompactness, Siberian Math. J. 38 (1997), no. 5, 926-928; translated from Sibirsk. Mat. Zh. 38 (1997), no. 5, 1071-1073, ii. MR 1486012

[21] N. A. Erzakova, On measure-compact operators, Russian Math. (Iz. VUZ) 55 (2011), no. 9, 37-42; translated from Izv. Vyssh. Uchebn. Zaved. Mat. 2011, no. 9, 44-51. MR 2931774.

[22] R. Gorenflo and B. Hofmann, On autoconvolution and regularization, Inverse Problems 10 (1994), no. 2, 353-373. MR 1269013

[23] G. Gripenberg, Periodic solutions of an epidemic model, J. Math. Biol. 10 (1980), no. 3, 271-280. MR 0599811.

[24] S. Hu, M. Khavanin and W. Zhuang, Integral equations arising in the kinetic theory of gases, Appl. Anal. 34 (1989), no. 3-4, 261-266. MR 1387174.

[25] P. Kolwicz, K. Leśnik and L. Maligranda, Pointwise products of some Banach function spaces and factorization, J. Funct. Anal. 266 (2014), no. 2, 616-659. MR 3132723

[26] M. A. Krasnosel'skiı̌ and Ja. B. Rutickiı̌, Convex functions and Orlicz spaces, P. Noordhoff, Groningen, 1961. MR 0126722

[27] T. Kuczumow, Fixed point theorems in product spaces, Proc. Amer. Math. Soc. 108 (1990), no. 3, 727-729. MR 0991700

[28] A. Leonard and T. W. Mullikin, Integral equations with difference kernels on finite intervals, Trans. Amer. Math. Soc. 116 (1965), 465-473. MR 0188735

[29] T. W. Mullikin, A nonlinear integrodifferential equation in radiative transfer, J. Soc. Indust. Appl. Math. 13 (1965), 388-410. MR 0185408

[30] E. Nursultanov and S. Tikhonov, Convolution inequalities in Lorentz spaces, J. Fourier Anal. Appl. 17 (2011), no. 3, 486-505. MR 2803945

[31] R. D. Nussbaum, A quadratic integral equation, Ann. Scuola Norm. Sup. Pisa Cl. Sci. (4) 7 (1980), no. 3, 375-480. MR 0597547

[32] R. O'Neil, Convolution operators and $L(p, q)$ spaces, Duke Math. J. 30 (1963), 129-142. MR 0146673

[33] R. O'Neil, Fractional integration in Orlicz spaces. I, Trans. Amer. Math. Soc. 115 (1965), 300-328. MR 0194881.

[34] L. B. Rall, Quadratic equations in Banach spaces, Rend. Circ. Mat. Palermo (2) 10 (1961), 314-332. MR 0144184.

[35] W. Rudin, Functional analysis, second edition, McGraw-Hill, New York, 1991. MR 1157815.

[36] I.A. Rus, An abstract point of view for some integral equations from applied mathematics, in: Proceedings of the International Conference, University of the West, Timişoara, 1997, pp. 256-270. 
[37] R. A. Ryan, Introduction to tensor products of Banach spaces, Springer-Verlag, London, 2002. MR 1888309

[38] Schwabik, Abstract Perron-Stieltjes integral, Math. Bohem. 121 (1996), no. 4, 425-447. MR 1428144

[39] H. M. Srivastava and R. G. Buschman, Theory and applications of convolution integral equations, Kluwer, Dordrecht, 1992. MR 1205580

[40] C. A. Stuart, Existence theorems for a class of non-linear integral equations, Math. Z. 137 (1974), 49-66. MR 0348416

[41] C. Swartz, A bilinear Orlicz-Pettis theorem, J. Math. Anal. Appl. 365 (2010), no. 1, 332-337. MR 2585105

[42] C. Swartz, Weak compactness in abstract duality pairs, Adv. Anal. 2 (2017), no. 2, 135-142. https://doi.org/10.22606/aan.2017.22008

[43] C. Swartz, Abstract duality pairs in analysis, World Scientific, Hackensack, NJ, 2018. MR 3753609

[44] M. Väth, A compactness criterion of mixed Krasnoselskiü-Riesz type in regular ideal spaces of vector functions, Z. Anal. Anwendungen 18 (1999), no. 3, 713-732. MR 1718161.

[45] M. Väth, Volterra and integral equations of vector functions, Marcel Dekker, New York, 2000. MR 1738341

[46] M. Väth, Ideal spaces, Lecture Notes in Mathematics, 1664, Springer-Verlag, Berlin, 1997. MR 1463946

[47] C. Zhai and D. R. Anderson, A sum operator equation and applications to nonlinear elastic beam equations and Lane-Emden-Fowler equations, J. Math. Anal. Appl. 375 (2011), no. 2, 388-400. MR 2735529

[48] X. Zhang, L. Liu and Y. Wu, Fixed point theorems for the sum of three classes of mixed monotone operators and applications, Fixed Point Theory Appl. 2016, Paper No. 49, 22 pp. MR 3500979

\author{
Kinga Cichoń \\ Institute of Mathematics, Faculty of Control, Robotics and Electrical Engineering, Poznan \\ University of Technology, Piotrowo 3A, 60-965 Poznań, Poland \\ kinga.cichon@put.poznan.pl \\ Mieczystaw Cichoń ${ }^{凶}$ \\ Faculty of Mathematics and Computer Science, A. Mickiewicz University, Umultowska 87, \\ 61-614 Poznań, Poland \\ mcichon@amu.edu.pl \\ Mohamed M. A. Metwali \\ Department of Mathematics, Faculty of Sciences, Damanhour University, Egypt \\ m.metwali@yahoo.com
}

Received: September 6, 2018

Accepted: August 6, 2019 